\title{
Acetylation of GATA4 on Lysine Residue K313 Promotes Osteoblastic Cells Grovth
}

\author{
Wenjun You Lijuan Song Kun Wang
}

Department of Endocrinology, Jining No. 1 People's Hospital, Jining, China

Key Words

Gata4 $\bullet$ Osteoporosis (OS) $\bullet$ HFOB1.19 cells $•$ Acetylation

\section{Abstract}

Background/Aims: GATA4, a protein related to whose acetylation is essential for cardiac defe impacts of GATA4 acetylation on osteoporosis and $293 T$ cells was detected after immunoprecipitation was conducted the modulation of GATA4 acetylation, transcriptional activity of GAT Furthermore, hFOB1.19 cells acetylation-defective mut? as a pivotal event in $\mathrm{hFO}^{\prime}$ site was on lysine residu rather than by $\mathrm{HDA}$-2 5 .

\section{9 cells.} ity ano the presence or absence of cycloheximide. $C$ inhibitors (TSA and SAHA). COas hine which HATs and HDACs was involved in as and to identify the acetylation site. The psults: As a result, GATA4 acetylation was identified 4 can be acetylated by P300/CBP, and the acetylation 13. B^ides, the acetylation of GATA4 can be impaired by HDAC1, cetylation contributed to the stability and transcription of GATA4. Moreov acetyration activated CCND2 transcription, and mutation of GATA4 on K-313 reduce al and increased a mitochondria-dependent apoptosis in hFOB1.19 cells. Cond rion un suggest that GATA4 exists as an acetylated protein in hFOB1.19 cells. A tion ates the stability and transcription of GATA4, and activates CCND2 tonscrip which may explain the growth-promoting functions of GATA4 in hFOB1.19 cells. steoporosis (OS) is defined as low bone mineral density caused by altered bone ostructure leading to skeletal fragility and fracture [1]. OS is one of these serious ealth problems, affecting millions of people worldwide [2, 3]. Under normal physiological conditions, osteoblasts are responsible for the formation of new bone in the developing skeleton and during the process of bone remodeling [4]. Recently, therapeutic strategies for OS attempt to promote bone formation by increasing the number or activity of mature

W. You and L. Song contributed equally to this work. 


\section{Cellular Physiology Cell Physiol Biochem 2018;46:269-278

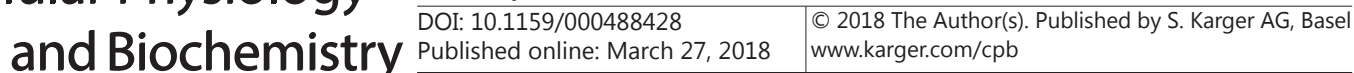 \\ You et al.: Role of GATA Acetylation in Osteoporosis}

osteoblasts or by preventing their apoptosis [5]. There is a need for therapy that target osteoblastic cells to increase their survival.

GATA4 is the founding member of the GATA family of zinc finger transcription factors, which consists of 6 members, GATA1 to GATA6 [6]. GATA4 is well known for its central role in embryogenesis [7], and in myocardial differentiation and function [8]. Besides, this prote is thought to be necessary for testicular development [9]. Mutations in this gene have bee associated with various diseases. For the selected examples, GATA4 mutations/deletion are causes of neonatal or childhood-onset diabetes with or without exocrine insufficie [10]. GATA4 genetic mutations are significantly associated with congenital part dis risk $[11,12]$. In terms of OS, GATA4 has been previously identified as a pion estrogen receptor in osteoblasts [13]. Further investigation demonstrated that $k$ GATA4 specifically in osteoblasts leads to embryonic and/or perinatal letb vivo evidence that GATA4 is essential for bone mineralization [14]. Besidc reported to be a negative regulator in osteoblast differentiation possibly Run2 [15], and bone sialoprotein (Bsp) [16].

Chromatin structure is determined by histone proteins, whic of covalent modifications that influence chromatin archit and g. of covalent modifications that influence chromatin archit and go voression [17].
Acetylation is certainly the best characterized post-t
mod ation of both chromatin accessibility by modulating histone function, an fy regulation of nonhistone proteins. Accruing studies indicated that histone aco nar deacetylation have many links to congenital cardiac abnormaliti 8], immun sponse [19], epilepsy [20, 21], and multiple kind of cancers, like ovarian er [22], go cancer [23], colorectal cancer [24], and lung cancer [25]. The balance be istone acetylation and deacetylation is maintained by the equilibrium of two kind nzy histone acetyltransferases (HATs) and histone deacetylases (HDACs), that HATs res wble for histone acetylation while the duty of HDACs is deacetylation [26]

A previous study has reported ay $T$ is able to acetylate GATA4, resulting in enhanced DNA binding and transcrip $y$ [27]. In transgenic mice, HAT in the heart induces GATA4 acetylation ? dio 28,29$]$. These authors demonstrated an essential role of GATA4 ac ion in cardiac defects; however, the functional impacts of GATA4 acetylation on OS, thowledge, have not been previously revealed. Here, we explored how GATA4 tylated aman osteoblastic hFOB1.19 cells, and examined the functions of GATA4 acet ion g the growth of hFOB1.19 cells.
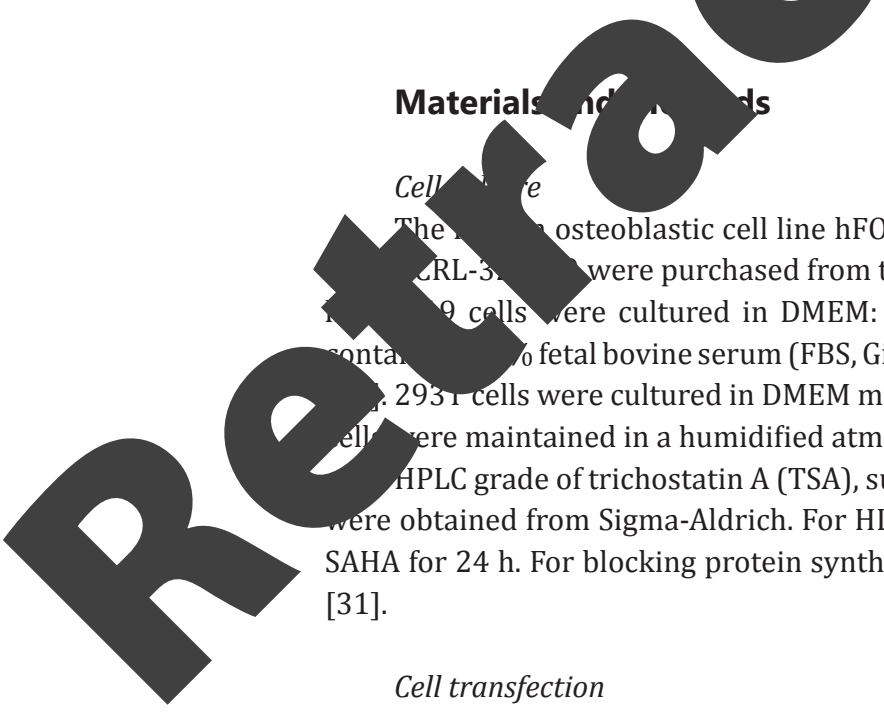

osteoblastic cell line hFOB1.19 (CRL-11372 ${ }^{\mathrm{Tm}}$ ) and the human embryonic kidney cell line
were purchased from the American Type Culture Collection (ATCC, Manassas, VA, USA).
APLC grade of trichostatin A (TSA), suberoylanilide hydroxamic acid (SAHA) and cycloheximide (CHX)

obtained from Sigma-Aldrich. For HDAC inhibition, cells were treated with 20, 40, and 80 nM TSA or SAHA for $24 \mathrm{~h}$. For blocking protein synthesis, cells were treated with $5 \mathrm{mg} / \mathrm{kg}$ CHX for the indicated time [31].

Cell transfection

Full-length GATA4 was generated by PCR and cloned into pc-DNA3.1-Flag vectors (Invitrogen, Carlsbad, CA, USA) to construct Flag-GATA4 vector. CBP, PCAF, P300, and GCN5 were sub-cloned into pcDNA3.1-Myc 


\section{Cellular Physiology Cell Physiol Biochem 2018;46:269-278 \begin{tabular}{l|l} 
and Biochemistry Published 10.1159/000488428 & $\begin{array}{l}\text { () 2018 The Author(s). Published by S. Karger AG, Basel } \\
\text { www.karger.com/cpb }\end{array}$
\end{tabular}}

You et al.: Role of GATA Acetylation in Osteoporosis

(Invitrogen). HDAC1 to HDAC5 were sub-cloned into pcDNA3.1-HA vectors, which were constructed by inserting a copy of the HA epitope tag (YPYDVPDYA) into the pcDNA3.1 vector (Invitrogen).

The position of lysines (K) 247, 257, 301, 313, 320, 322, 324, 328, 330, 366, 405, and 424 in GATA4 were substituted to arginines (R). The mutated GATA4 sequence was cloned into pc-DNA3.1-Flag vectors. Point mutations in GATA4 were introduced by generating two overlapping fragments by PCR and were verifi by sequencing. For GATA4 silence, cells were transfected with siRNA against GATA4 (RiboBio, Guangzho China). Control cells were transfected with empty vectors or non-targeting scrambled controls. Transfection was performed by using Lipofectamine 2000 (Invitrogen) according to the manufacturer's protocol. transfected cells were subjected to $1.0 \mu \mathrm{g} / \mathrm{mL}$ neomycin for stable cell selection.

\section{Co-immunoprecipitation (Co-IP) and western blot}

The protein used for co-immunoprecipitation and immunobloting were extracte RIPA lysis buffer (Beyotime Biotechnology, Shanghai, China). The whole-cell lysates u $200 \mathrm{~g}$ for $10 \mathrm{~min}$ at $4^{\circ} \mathrm{C}$, and the supernatant was incubated with anti-GATA4 (Cell Danvers, MA, USA), anti-IgG (Santa Cruz Biotechnology, Santa Cruz, CA), anti-Flag (S`? and protein-A sepharise (Zymed Laboratories, South San Francisco, CA, USA), an

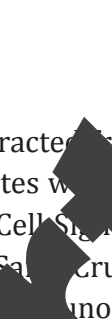
acetylated-Lys (New England Biolabs, Beverly, MA, USA), anti-GATA 1 Signalin,

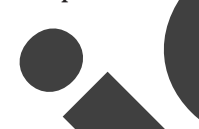
(Abcam, Cambridge, MA), and anti-HA (BioVision, Mountain View, were separate by SDS-PAGE and transferred into PVDF membranes the secondary antibody and the bands were visualized using chemil MA, USA).

For western blotting, the following antibodies we anti-GATA HDAC1 (Abcam), anti-cleaved caspase-3 (Abcam), anti- $\mathrm{B}$ and anti-GAPDH (Abcam).

\section{Promoter reporter and dual luciferase ass}

293T cells were seeded in 24-well pl 05 cens /well) for adherence, and transfected with Lipofectamine 2000 (Invitrogen) in antibi $48 \mathrm{~h}$. The biological activity of luciferase reporter construct was assessed Reporter Assay System (Promega, Madison, WI, USA). The relative luciferase activ normanen to that of firefly luciferase.

\section{m), anti-Bax (Abcam), anti-Bcl-2 (Abcam),}

\section{Cell viability}

After correspondingly 5000 cells/well for adheren were washed twic were incubated measured b

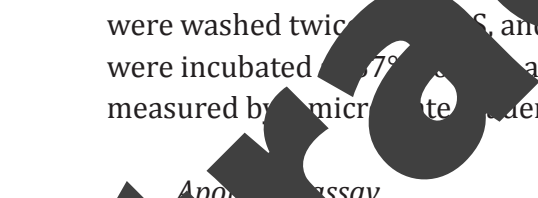

sfecti hFOB1.19 cells were planted in 96-well plates with a density of 3 and 4 days of culturing, the culture medium was removed, cells antuzo ML MTT (Sigma-Aldrich) solution was added into each well. The plates after which $10 \mu \mathrm{L}$ DMSO was added to dissolve crystal. The absorbance was er (Bio Rad Laboratories, Hercules, CA, USA) at a wavelength of $570 \mathrm{~nm}$.

Ce Ant U-FITC Apoptosis Staining/Detection Kit from Abcam (Cambridge, MA, USA) was utilized sent w $k$, according to the manufacturer's protocols. hFOB1.19 cells were seeded in 6-well plates $5 \times 10^{5}$ cells/well for adherence. After the indicated transfection, cells in each sample colrected by trypsin digestion and centrifugation, and were resuspended in $200 \mu \mathrm{L}$ of binding buffer ging $5 \mu \mathrm{L}$ Annexin V-FITC solution and $5 \mu \mathrm{L}$ PI. The samples were incubated in the dark for $30 \mathrm{~min}$ at ter which cells were analyzed by the FACS can (Beckman Coulter, Fullerton, CA). The early apoptotic were calculated, which characterized by FITC-positive and PI-negative.

\section{Statistical analysis}

All experiments were repeated three times. Data were expressed as mean \pm SD. Differences between groups were analyzed by one-way analysis of variance (ANOVA) with Duncan procedure in SPSS version 19.0 program (SPSS Inc., Chicago, IL, USA). A P-value of $<0.05$ was considered to indicate a statistically significant result. 
Results

GATA4 acetylation was observed in hFOB1.19 cells, and acetylation contributed to GATA4 stability

hFOB1.19 cells were subjected to various dose of HDAC inhibitors (TSA and SAHA) for 24 $h$. As a result, we found that protein level expressions of GATA4 were significantly increased by TSA and SAHA in a dose-dependent fashion $(P<0.01$ or $P<$ 0.001 , Fig. 1A), indicating GATA4 acetylation promoted GATA4 expression. This observation was confirmed in 293T cells, that protein expressions of GATA4 in 293T cells pre-transfected with Flag-GATA4 were also increased by TSA or SAHA treatment $(P<$ 0.01 or $P<0.001$, Fig. 1B). Then, acetylation of GATA4 in 293T cells were detected by immunoblot with anti-Acetylated-Lys post-transfection with FlagGATA4 vector or Flag vector. As results shown in Fig. 1C, vi acetylation signal was foun cells transfected with $\mathrm{F}$ $\mathrm{TA} 4$, rather than in cel fected with the Flag vec was conducted in hEOB1. to determine endogenous $C$ cells upon

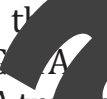
showed , ac
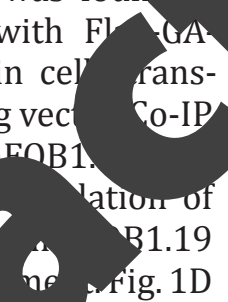
ation of B1.19 ace on signal
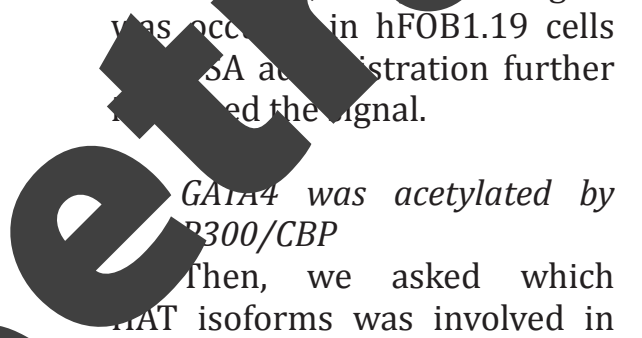
GAtr4 was acetylated by 300/CBP Then, we asked which isoforms was involved in mediating GATA4 acetylation in hFOB1.19 cells. To do this, 293T cells were co-transfected with Flag-GATA4 and expression vectors encoding $\mathrm{CBP}, \mathrm{PCAF}$, P300 or GCN5. Co-IP results revealed that co-transfection of Flag-GATA4 and Myc-CBP,

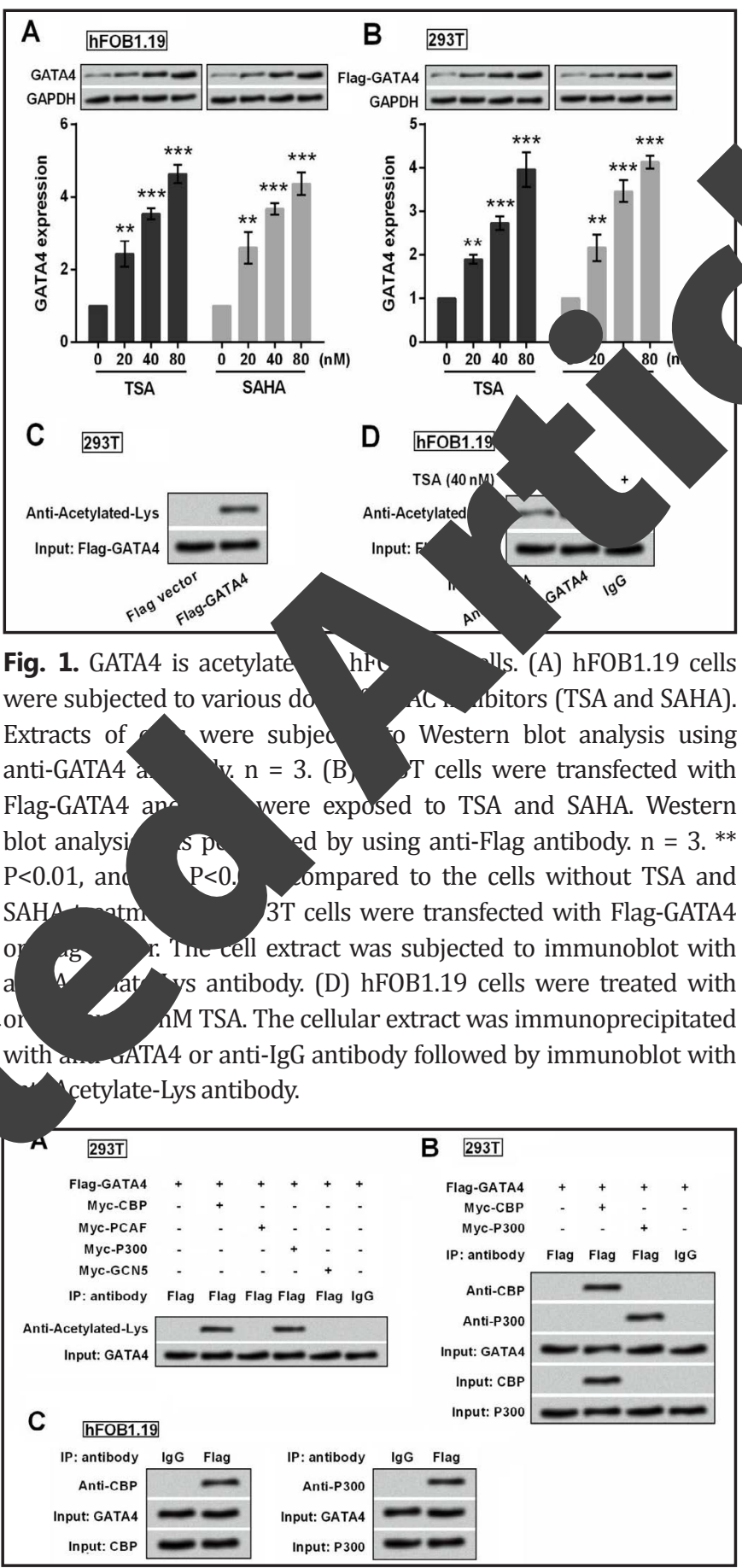

Fig. 2. Acetylation of GATA4 by P300/CBP. (A) 293T cells were transfected with Flag-GATA4 and expression vectors encoding CBP, PCAF, P300 or GCN5. The cellular extract was immunoprecipitated with antiFlag or anti-IgG antibody followed by immunoblot with anti-AcetylateLys antibody. (B) 293T cells were transfected with Flag-GATA4 and expression vectors encoding CBP or P300. The extract was immunoprecipitated with anti-Flag or anti-IgG antibody followed by immunoblot with anti-CBP and anti-P300 antibody. (C) The extract from hFOB1.19 cells was subjected with immunoprecipitation using anti-Flag or antiIgG antibody followed by immunoblot with anti-CBP and anti-P300. 


\section{Cellular Physiology and Biochemistry}

as well as co-transfection of Flag-GATA4 and Myc-P300 resulted in visible acetylation signals (Fig. 2A), indicating GATA4 acetylation was mediated by CBP and P300, rather than by PCAF and GCN5. The interaction between GATA4 and P300/CBP was determined by Co-IP in 293T cells. Results in Fig. 2B showed that GATA4 specifically interacted with CBP and P300, but not with the IgG control. The phenomenon was also observed in hFOB1.19 cells, that GATA4 could interact with CBP and P300 rather than IgG at an endogenous level (Fig. 2C).

The site of acetylation occurred on lys-313 in GATA4

To determine which region in GATA4 was acetylated by P300/CBP, different K-point mutation

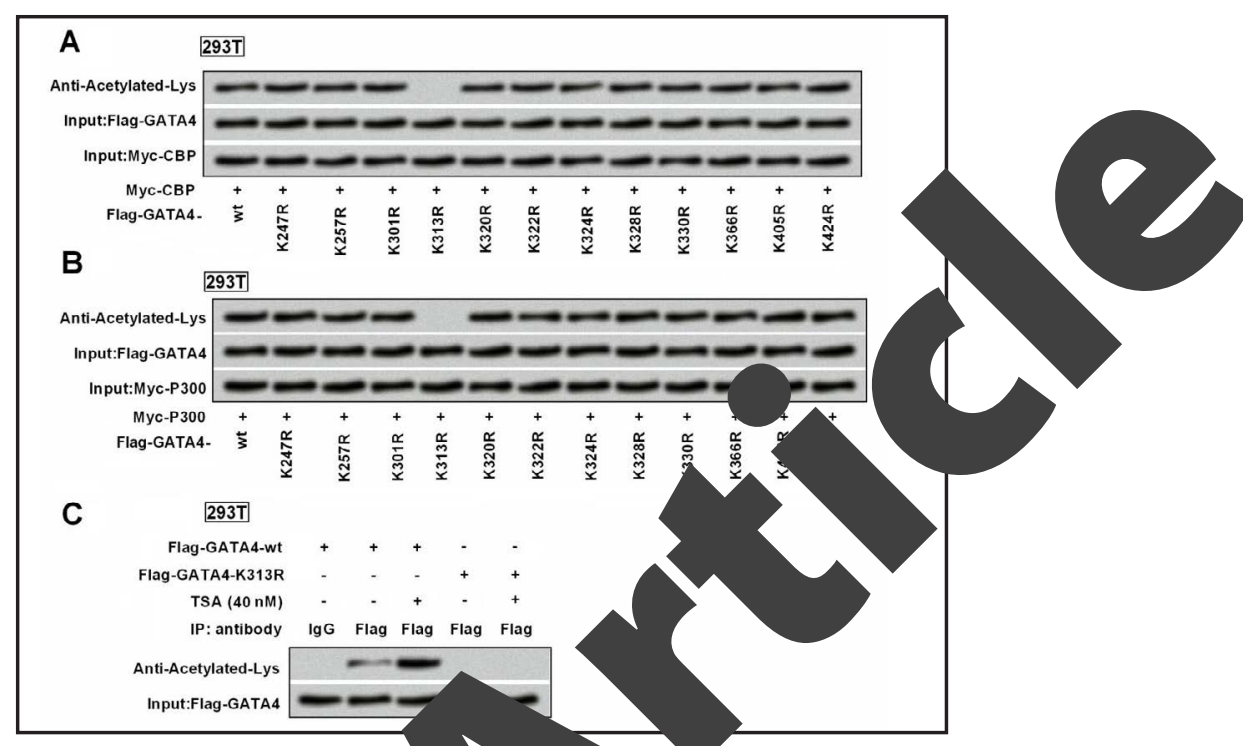

Fig. 3. Identification of GATA4 vlaty (A) 293T cells were cotransfected with Myc-CBP and e the indicated posi nn of lysines vere substituted to arginines
(R). Cell extract Is of GATA4, in which Lys antibody. (B) 29. "s were corransfected with Myc-P300 and expression vecto co utated GATA4, after which immunoblot was performed. 2931 were transfected with Flag-GATA4wt (wild trme) TA4-K313R (mutated at lys-313). The transfer yerumured with or without $40 \mathrm{nM}$ TSA for $24 \mathrm{~h}$, after v e ract was collected for immunoprecipitation using fylate antibody followed by immunoblot using antiwas designed ( $\mathrm{K}$ mutat to $\mathrm{R}$ ), and then the mut Fig. 3A showed that, exc all other groups pact-tra GATA4 ther with Myc-CBP was transfected into 293T cells. region in GATA the P300 was the same as CBP, that no acetylation signal was observed in ce cted with Myc-P300 and Flag-GATA4-K313R (Fig. 3B). There data strongly ey ence ho pond P300 directly interacte with the region of lys-313 in GATA4. Fig. 3C ed th could not induce the acetylation of GATA4 when Flag-GATA4-K313R a into 293T cells, further implying the site of acetylation occurred on lys-313

\section{lation of GATA4 by HDAC1}

To uetermine which HDAC is specially involved in the acetylation of GATA4, 293T cells co-transfected with Flag-GATA4, Myc-CBP and expression vectors encoding HDAC1 SAC5. Co-IP results revealed that acetylation signal induced by GATA4-CBP interaction as reduced by transfection with HA-HDAC1, rather than other HA-HDACs (Fig. 4A). The interation between GATA4 and HADC1 was then confirmed in 293T (Fig. 4B) and hFOB1.19 cells (Fig. 4C).

\section{Acetylation promoted the transcriptional activity of GATA4}

CHX is widely used as an inhibitor of protein synthesis. Herein, 293T cells were transfected with Flag-GATA4 or transfected with Flag-GATA4 plus Myc-CBP, and then subjected with CHX for $24 \mathrm{~h}$. Down-regulated expression of GATA4 was observed in Flag-GATA4 transfected cells prolonged exposure to CHX (Fig. 5A). However, the down-regulations of GATA4

\section{KARGER}


induced by CHX exposure were not that remarkable when cells were cotransfected with Flag-GATA4 and MycCBP. Considering that the synthesis of GATA4 protein was sharply declined at $8 \mathrm{~h}$ of CHX exposure, 3, 6, and $9 \mathrm{~h}$ were selected for the CHX-treating conditions for use in the following experiments. Fig. 5B showed that, the down-regulations of GATA4 induced by CHX exposure were accelerated by co-transfection with Flag-GATA4 and HA-HDAC1 relative to transfection with Flag-GATA4 alone. Fig. 5C indicated that, the protein level expressions of GATA4 in 293T cells transfected with Flag-GATA4-wt or Flag-GATA4-K313R were reduced by prolonged CHX exposure.

GATA4 is known to activate CCND2 promoter [32]. Herein, we overexpressed wt or mutated GATA4 and CBP or HDAC1 in 293T cells, and measured CCND2 promoter activity, to detect whether GATA4 acetylation will impact the targeting effects between GATA4 and CCND2. As results shown in Fig. 5D and 5E, GATA4 and CBP together synergistically the CCND2 promoter activi HDAC1 antagonized GAT -ino ile

Fig. 5. Enhanced transcr ity of GATA4 by ity of GATA4 by cells were trans or transfected w plus Myc$\mathrm{CBP}$, and the bje r. $\mathrm{CHX}$ for $24 \mathrm{~h}$. Protein sions $\mathrm{A} 4$ were probed body. (B) 293T cells were cted th ag-GATA4 and/or HAndnubsected with CHX for $9 \mathrm{~h}$. sions of GATA4 were probed

inti-Flag antibody. (C) 293T cells transc' vith Flag-GATA4-wt or Flag-GATA4oR were subjected with CHX for $9 \mathrm{~h}$. rotein expressions of GATA4 were probed by anti-Flag antibody. (D) 293T cells were transfected with Flag-GATA4-wt/FlagGATA4-K313R and Myc-CBP. The CCND2 promoter activity was then detected. $\mathrm{n}=3$.

(E) 293T cells were transfected with Flag-

GATA4-wt/Flag-GATA4-K313R and Myc-HDAC1, after which CCND2 promoter activity was detected. $\mathrm{n}=3$. ${ }^{*}$ $\mathrm{P}<0.05,{ }^{* *} \mathrm{P}<0.01$, and ${ }^{* * *} \mathrm{P}<0.001$ compared to control group. Ns, no significance, $\# \mathrm{P}<0.05$, and \#\# $\mathrm{P}<0.01$ compared to the indicated group.

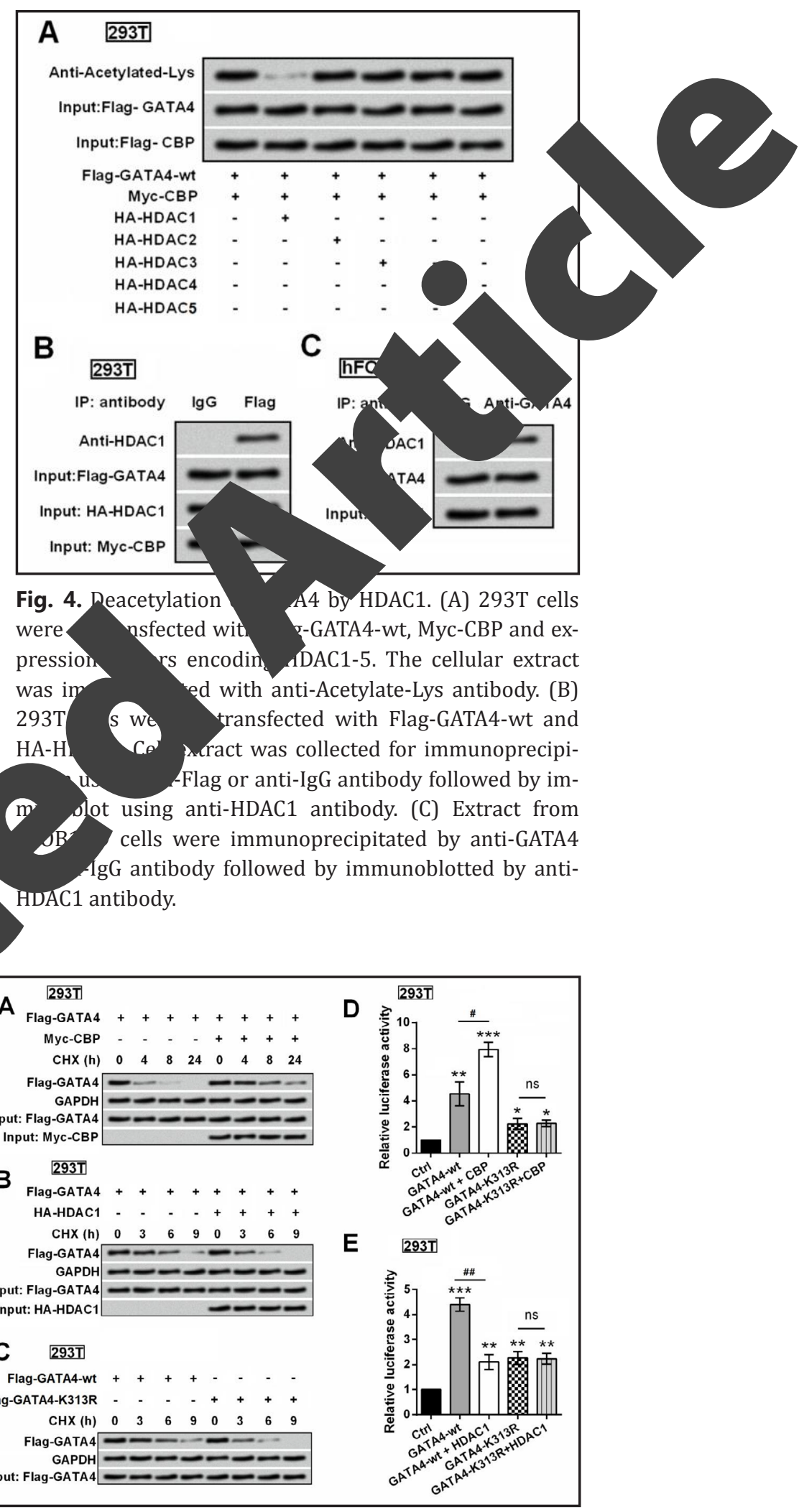


Fig. 6. Acetylation-defective mutant of GATA4 reduces hFOB1.19 cells growth. (A) hFOB1.19 cells were transfected with siGATA4, oeGATA4wt or oeGATA4-K313R. Western blot was performed to detect GATA4 expression to ensure the transfection efficiency. (B) Viability of hFOB1.19 cells after siGATA4 transfection. $\mathrm{n}=$ 3. (C) Viability of hFOB1.19 cells after oeGATA4-wt or oeGATA4-K313R transfection. $\mathrm{n}=3$. (D) Apoptotic rate of hFOB1.19 cells after siGATA4, oeGATA4-wt or oeGATA4-K313R transfection. $n=3$. (E) Protein levels of cleaved caspase-3, Bid, Bax, and Bcl-2 in hFOB1.19 cells after the indicated transfection. * $\mathrm{P}<0.05$, ** $\mathrm{P}<0.01$, and $* * * \mathrm{P}<0.001$ compared to control group. \# $\mathrm{P}<0.05$ comparison between oeGATA4-wt and oeGATA4-K313R groups.

increase of CCND2 promoter activity. Howe promoter activity when lys-313 in GATA4 was
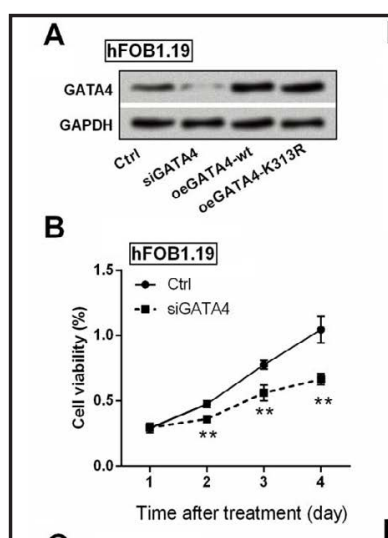

C
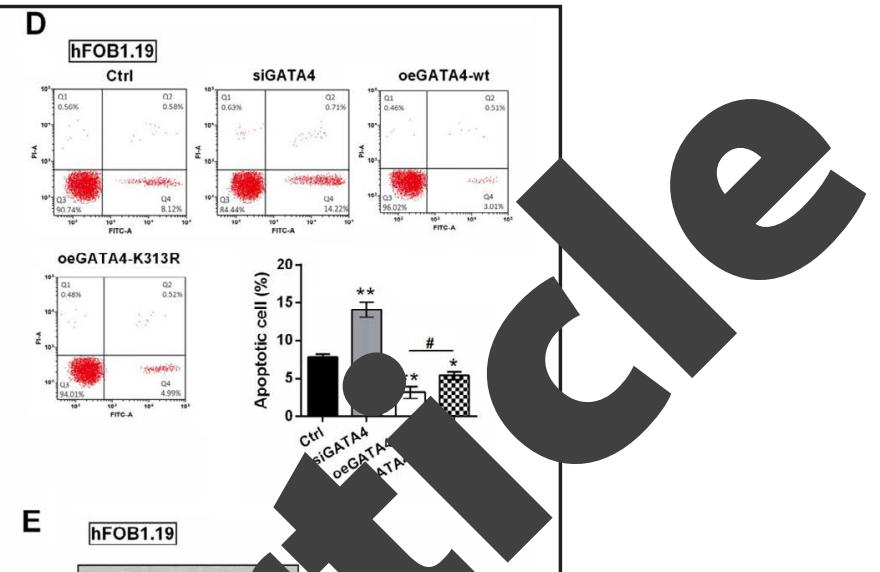
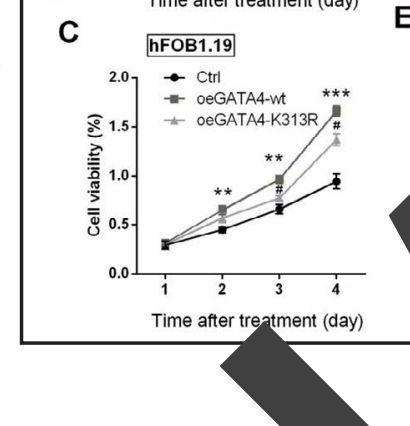

CBI HDAC1 could not affect CCND2 roded $(P>0.05)$.

GATA4 mutation at K313 reduced ef. hi-promoting activities of GATA4 on hFOB1.19
cells
In order to investigate the growth of hFOB1.19 ce transfected into cells. W and results showed that transfection, while was i MTT assay result: -1 icato by siGATA4 $(P \quad$ reased by oeGATA4 $(P<0.01$ or $P<0.001)$, while oeGATA4-induced increased of 1 was neutralizated by GATA4 mutation at lys-313 (Fig. 6B and 6C). Flow ection results showed that the apoptotic cell rate was significantly increas siGA $P<0.01)$, decreased by oeGATA4 $(P<0.01)$; unsuprisingly oeGATA4$j$ Jund of cell apoptosis was neutralizated by GATA4 mutation at lys-313 (Fig. veste $t$ analytical results further confirmed the alleviating effect of GATA4 point non apoptosis. As shown in Fig. 6E, up-regulations of cleaved caspase-3, Bid and Bax, egulation of Bcl-2 were observed in siGATA4 transfected cells; oeGATA4 affected

e protein expressions resulted in a completely opposite impacts, and these alterations ed by oeGATA4 were alleviated by GATA4 mutation at lys-313.

\section{Discussion}

Bone remodeling is controlled by osteoblasts and osteoclasts, the imbalance between osteoblasts and osteoclasts leads to osteopetrosis or OS by changing bone mass. Recently, studies have demonstrated the necessity of GATA4 in osteoblasts, that GATA4 regulates osteoblast differentiation $[15,16]$, which contributes in estrogen-independent mineralization [14]. Here we identified GATA4 acetylation as a pivotal event in human osteoblastic hFOB1.19 cells. Specifically, we observed that GATA4 in hFOB1.19 cells was acetylated by P300/CBP, and the acetylation site was on lys-313; besides, the acetylation of KARGER 


\section{Cellular Physiology Cell Physiol Biochem 2018;46:269-278 \begin{tabular}{l|l} 
and Biochemistry POI: 10.1159/000488428 & $\begin{array}{l}\text { (c) } 2018 \text { The Author(s). Published by S. Karger AG, Basel } \\
\text { www.karger.com/cpb }\end{array}$
\end{tabular} \\ You et al.: Role of GATA Acetylation in Osteoporosis}

GATA4 could be impaired by HDAC1, rather than by HDAC2-5. GATA4 acetylation contributed to the transcriptional activity of GATA4. What's more, mutation of GATA4 on lys-313 reduced the growth-promoting activities of GATA4 on hFOB1.19 cells, suggesting the acetylation of GATA4 on lys-313 promoted osteoblastic cells growth in vitro.

Human HATs can be divided into several large families: MYST (TIP60, MOZ/MYST MORF/MYST4, HBO1/MYST2, MOF/MYST1), P300/CBP (P300, CBP), and GNAT (GCNs, PCAF, ELP3) [33]. These HATs have different lysine specificities, and remarkably, P300/CBP acetylates all four core histones in nucleosomes [34]. A previous study has revealed P300 is able to acetylate lys-311, $-318,-320$ and -322 of GATA4 in myocardial addition to P300, CBP possesses HAT activity and P300/CBP is able to acety two highly conserved, lysine-rich motifs near the zinc finger domains $[35,36]$ for the first time observed that GATA4 was acetylated by P300/CBP on ly rather than by PCAF or GCN5.

In contrast to HAT, HDACs mediate histone deacetylation. Accordi with the yeast counterparts, HDACs are grouped into five sub-familiea and 8), class IIa (HDAC4, 5, 7, and 9), class IIb (HDAC6 and HDAC10), III (sirtuins) and class IV (HDAC11) [37]. In the current study, GATA4 co-in with CBP was almost abolished by HDAC1 except by HDAC2-5, suggest by CBP can be impaired by HDAC1.

Acetylation has been mentioned to regulate the funct such as DNA-binding activity, protein interactions at multiple levels transactivation activity [38]. In the current stu o inhibito f HDAC activity (TSA and SAHA), which cause accumulation of acetylated. species in a variety of mammalian cell lines [39, 40], were used to promote the cyla of GATA4. As a result, the protein expressions of GATA4 were increased by Ts nd suggesting GATA4 protein has in some manner maintained its stabilit cetylation. The accumulated GATA4 due to its stability promotes GATA4' 110 , for example senescence, a terminal arrest of proliferation triggered by variou. ar resses including dysfunctional telomeres, DNA damage, oxidative stre nutations [41]. On the other hand, our data from Co-IP suggested that G ccumuration can be enhanced by histone acetylation via triggering GATA4 transcri ont. ervation was confirmed with the widely accepted viewpoint that histone a lation ociated with an "open" chromatin configuration and active transcription [42

It is well-knowm that by the CCND2 Mans. A previous study has pointed out that GATA4 could activate CCND2 promo activity, w GA ated at lys-313 showed low transactivation potential. Considering that GA cety implicated in the transcription of CCND2, we speculate that it will g in hFOB1.19 cells growth. To test this hypothesis, the viability and apoptosis B1.1 $\quad s$ were detected. Our data evidenced that GATA4 acetylation at lys-313 may forinu easing cell viability, and repressing apoptosis in a mitochondria-dependent mutation at lys-313 decreased the ratio of Bcl- 2 to Bax, increased Bid expression sequentially activated caspase-3. This finding is consistent with the studies of Yanazume 28]. and Trivedi et al [43]., of which GATA4 acetylation was reported to be implicated adiac myocyte growth. Although, the mechanisms have not been fully resolved, one of 1e putative mechanisms of GATA4 acetylation for growth may be a functional modulation of CCND2 or other transcription factors related to cell growth.

\section{Conclusion}

In summary, our data suggest that GATA4 exists as an acetylated protein in osteoblastic hFOB1.19 cells. GATA4 was acetylated by P300/CBP on lysine residue K313. Acetylation regulates the stability and transcription of GATA4, and activates CCND2 transcription, may be via which histone posttranslational modification of GATA4 promoted hFOB1.19 cells 


\section{Cellular Physiology Cell Physiol Biochem 2018;46:269-278 \begin{tabular}{l|l} 
and Biochemistry Published online: March 27, 2018 & $\begin{array}{l}\text { (c) } 2018 \text { The Author(s). Published by S. Karger AG, Basel } \\
\text { www.karger.com/cpb }\end{array}$
\end{tabular}}

You et al.: Role of GATA Acetylation in Osteoporosis

growth. The findings in this study provide a possibility for use GATA4 as an efficient targeted therapy in the control of OS. However, more effort is required to evaluate the potential usage of GATA4-based target in OS.

\section{Disclosure Statement}

No conflict of interests exists.

\section{References}

1 Porter JL, Bhimji SS: Osteoporosis; in StatPearls. Treasure Island (FL), StatPearls Publishing LL p.^pp.

-2 Svedbom A, Hernlund E, Ivergard M, Compston J, Cooper C, Stenmark J, McCloskey E JA: Osteoporosis in the European Union: a compendium of country-specific reports. 2013;8:137.

-3 Wade SW, Strader C, Fitzpatrick LA, Anthony MS, O’Malley CD: Estimating pre examples from industrialized countries. Arch Osteoporos 2014;9:182

4 Ottewell PD: The role of osteoblasts in bone metastasis. J Bone Or

5 Marie PJ, Kassem M: Osteoblasts in osteoporosis: past, emerging, Endocrinol 2011;165:1-10.

6 Ferreira R, Ohneda K, Yamamoto M, Philipsen S: GATA1 function, a p hematopoiesis. Mol Cell Biol 2005;25:1215-1227.

7 Geraud C, Koch PS, Zierow J, Klapproth K, Busch K, Singh S, Sticht C, Breitkopf-Heinlein K, Richter K, Karpr Augustin HG, Schledzewski K, Goerdt S: GATA4-dep controls liver development and embryonic hematd Jiang XY, Feng YL, Ye LT, Li XH, Feng J, Zhang MZ, Sh Gata 4 and Tbx5 by Nicotine-Mediated DN 2017;8:290-304.

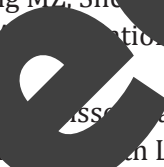
sis. Jy V, Leibing mory A, Ulbrich F, Diett M, 4, Pihlajaniemi T, Arnold B, Rodewald HR, specific endothelial differentiation rest 2017;127:1099-1114. sler M, Li Y, Geng YJ, Yu XY: Inhibition of ocardial Differentiation. Stem Cell Reports

an Zanten MA, van Herwaarden AE, HulsbergenLB, Sweep F, Claahsen-van der Grinten HL: GATA
-9 Engels M, Span PN, Mitchell RT Heyvel van de Kaa CA, Oosterwijk E, transcription factors in testicr

\section{ngo} e Nancla nal restcumours. Endocr Connect 2017;6:866-875. tlle M, Flanagan SE, Borowiec M, Taplin CE, van Alfen-van der Miedzybrodzka Z, Deja G, Wlodarska I, Mlynarski W, Ferrer J, 4 mutrions are a cause of neonatal and childhood-onset diabetes. Diabetes

in $\mathrm{S}$ Iy KS, Thangaraj K, Banerjee SK: c.620C>T mutation in GATA4 is heart disease in South India. BMC Med Genet 2015;16:7.

12 Zhang YAi F, Zh J. Bssociations of GATA4 genetic mutations with the risk of congenital heart diseas neta Medicine (Baltimore) 2017;96:e6857.

13 Mi Carbon_quemes M, Bailey S, Anaya E, Corselli M, Peault B, Krum SA: GATA4 regulates estrogen ha-mediated osteoblast transcription. Mol Endocrinol 2011;25:1126-1136.

uemes cia AJ, Rigueur D, Runke S, Wang W, Zhao G, Mayorga VH, Atti E, Tetradis S, Peault B, Lyons K, ndn-Cârboni GA, Krum SA: GATA4 is essential for bone mineralization via ERalpha and TGFbeta/BMP J Bone Miner Res 2014;29:2676-2687.

Song1, Kim K, Kim JH, Lee YK, Jung HJ, Byun HO, Yoon G, Kim N: GATA4 negatively regulates osteoblast differentiation by downregulation of Runx2 BMB Rep 2014;47:463-468.

ong I, Jeong BC, Choi YJ, Chung YS, Kim N: GATA4 negatively regulates bone sialoprotein expression in osteoblasts. BMB Rep 2016;49:343-348.

Aronson BE, Rabello Aronson S, Berkhout RP, Chavoushi SF, He A, Pu WT, Verzi MP, Krasinski SD: GATA4 represses an ileal program of gene expression in the proximal small intestine by inhibiting the acetylation of histone H3, lysine 27 Biochim Biophys Acta 2014;1839:1273-1282.

18 Wu G, Nan C, Rollo JC, Huang X, Tian J: Sodium valproate-induced congenital cardiac abnormalities in mice are associated with the inhibition of histone deacetylase. J Biomed Sci 2010;17:16.

19 Cameron AM, Lawless SJ, Pearce EJ: Metabolism and acetylation in innate immune cell function and fate. Semin Immunol 2016;28:408-416.

-20 Detich N, Bovenzi V, Szyf M: Valproate induces replication-independent active DNA demethylation. J Biol Chem 2003;278:27586-27592. 


\section{Cellular Physiology and Biochemistry \\ Cell Physiol Biochem 2018;46:269-278 \\ \begin{tabular}{l|l}
\hline DOI: $10.1159 / 000488428$ & (c) 2018 The Author(s). Published by S. Karger AG, Basel
\end{tabular} \\ Published online: March 27, 2018 www.karger.com/cpb}

You et al.: Role of GATA Acetylation in Osteoporosis

-21 Eyal S, Yagen B, Sobol E, Altschuler Y, Shmuel M, Bialer M: The activity of antiepileptic drugs as histone deacetylase inhibitors. Epilepsia 2004;45:737-744.

-22 Pchejetski D, Alfraidi A, Sacco K, Alshaker H, Muhammad A, Monzon L: Histone deacetylases as new therapy targets for platinum-resistant epithelial ovarian cancer. J Cancer Res Clin Oncol 2016;142:1659-1671.

23 Choi JH, Kwon HJ, Yoon BI, Kim JH, Han SU, Joo HJ, Kim DY: Expression profile of histone deacetylase 1 in gastric cancer tissues. Jpn J Cancer Res 2001;92:1300-1304.

-24 Weichert W, Roske A, Niesporek S, Noske A, Buckendahl AC, Dietel M, Gekeler V, Boehm M, Beckers T, Denkert C: Class I histone deacetylase expression has independent prognostic impact in human colorecta cancer: specific role of class I histone deacetylases in vitro and in vivo. Clin Cancer Res 2008;14:1669-1

-25 Brodie SA, Li G, El-Kommos A, Kang H, Ramalingam SS, Behera M, Gandhi K, Kowalski J, Sic? Khuri Vertino PM, Brandes JC: Class I HDACs are mediators of smoke carcinogen-induced stabiliz and serve as promising targets for chemoprevention of lung cancer. Cancer Prev Res (Phila) 2 361.

26 Peserico A, Simone C: Physical and functional HAT/HDAC interplay regulates protein Biomed Biotechnol 2011;2011:371832.

27 Takaya T, Kawamura T, Morimoto T, Ono K, Kita T, Shimatsu A, Hasegawa K: Identif targeted acetylated residues in GATA4 during hypertrophic responses in card 2008;283:9828-9835.

28 Yanazume T, Hasegawa K, Morimoto T, Kawamura T, Wada H, Ma Cardiac p300 is involved in myocyte growth with decompensatec 3606.

29 Miyamoto S, Kawamura T, Morimoto T, Ono K, Wada H. Kawase Y, Ma K. Histone acetyltan A, Nishio R, Kita T, Hasegawa $\mathrm{K}$ : Histone acetyltransferase activity of p300 is req for the promo fleft ventricular remodeling after myocardial infarction in adult mice in vivo. Circ, 2006;113:6 90.

-30 Pan XW, Zhao XH: In vitro Proliferation and Anti-Apont. Hydrolysates towards Osteoblastic Cells (hFOB1.19

-31 Farber JL, Farmar R: Differential effects of cyclohex dose. Biochem Biophys Res Commun 1973;51.626-

-32 Anttonen M, Pihlajoki M, Andersson N, Ge RA, Heikinheimo M: FOXL2, GATA4, and apoptosis in ovarian granulosa ell "umd

-33 Gadhia S, Shrimp JH, Meier JK Probe Discovery; in (Sittamp C, Baell J, Bejcek B, Chung ' Iversen PW, Kahl SD, Kal the National Center for

-34 Ogryzko VV, Schiltz RL, CBP are histo 35 Blobel GA, ajp 1998.

\section{Hon attulainen S, Farkkila A, Unkila-Kallio L, Veitia} 2015;16:13908-13920.

Papain-Generated Casein and Soy Protein en on RNA synthesis as a function of ADeratively modulate gene expression, cell viability and PV ne 2014;9:e85545.

E, D Mstone Acetyltransferase Assays in Drug and Chemical

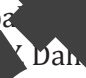
Coussens NP, Brimacombe K, Grossman A, Arkin M, Auld D, Austin Dah anaryan V, Foley TL, Glicksman M, Hall MD, Hass JV, Inglese J, C,et al. eas,_say Guidance Manual. Bethesda (MD), Eli Lilly \& Company and ncing 'sanslational Sciences, 2004, vol. p.^pp.

Howard BH, Nakatani Y: The transcriptional coactivators p300 and Cell 1996;87:953-959. and is required for erythroid differentiation. Proc Natl Acad Sci U S A

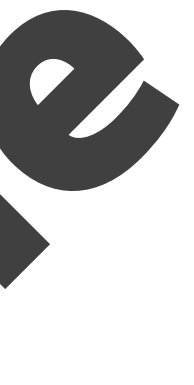

Hu Lau J, Kmm, Weiss MJ, Blobel GA: CREB-Binding protein acetylates hematopoietic transcription

Itor at functionally important sites. Mol Cell Biol 1999;19:3496-3505.

e Ruijte an Gennip AH, Caron HN, Kemp S, van Kuilenburg AB: Histone deacetylases (HDACs):

roch rization of the classical HDAC family. Biochem J 2003;370:737-749.

a F, Towatari M, Ozawa Y, Tomita A, Privalsky ML, Saito H: Functional regulation of GATA-2 by

acetyration. J Leukoc Biol 2004;75:529-540.

(1)

pshida M: [Potent and specific inhibition of mammalian histone deacetylase both in vivo and in vitro by

crichostatin A]. Tanpakushitsu Kakusan Koso 2007;52:1788-1789.

Richon VM, Emiliani S, Verdin E, Webb Y, Breslow R, Rifkind RA, Marks PA: A class of hybrid polar inducers of transformed cell differentiation inhibits histone deacetylases. Proc Natl Acad Sci U S A 1998;95:3003-

3007.

41 Kang C, Elledge SJ: How autophagy both activates and inhibits cellular senescence. Autophagy 2016;12:898-899.

42 Davie JR: The nuclear matrix and the regulation of chromatin organization and function. Int Rev Cytol 1995;162a:191-250.

43 Trivedi CM, Zhu W, Wang Q, Jia C, Kee HJ, Li L, Hannenhalli S, Epstein JA: Hopx and Hdac2 interact to modulate Gata4 acetylation and embryonic cardiac myocyte proliferation. Dev Cell 2010;19:450-459. 